\title{
A Study on Prevalence of Reproductive Tract Infections Amongst Women Attending in an Urban Clinic
}

\author{
Kabir Ahmed Khan ${ }^{1 *}$ \\ Sk Md. Jaynul Islam² \\ Shirin Akhter $^{3}$
}

${ }^{1}$ Commanding Officer BNS Patenga

Navy Hospital

Chittagong, Bangladesh.

${ }^{2}$ Department of Pathology

BNS Patenga

Navy Hospital

Chittagong, Bangladesh.

${ }^{3}$ Department of Gynae \& Obstetrics Marks Medical College \& Hospital Dhaka, Bangladesh.

\begin{abstract}
Introduction: One element of reproductive health that directly concerns human well being is reproductive tract infections (RTI). The magnitude of the problem of RTIs is a growing public health concern in developing countries like Bangladesh. Aim: A study was carried out in the out patient department of a busy urban clinic with an attempt to study the Reproductive Tract Infections (RTIs) . Materials and Methods: A descriptive type of cross sectional study was carried out in the Gynaecology OPD of an urban clinic at Dhaka, the capital of Bangladesh during the period from July 2007 to December 2007. The study population includes the women of reproductive age group (15-49 years). Among 1061 reported women 176 respondents were selected for the study with a systematic purposive sampling technique. Data were collected from prescription sheets of the concerned gynecologist, laboratory investigation reports and questionnaires used for interview. Results: Mean age of the respondents was 30.56+6.1. Among 176, 174 (98.86\%) were married. Depending on clinical symptoms $32(18.19 \%)$ were diagnosed as RTI, after clinical examinations 39 $(22.16 \%)$ and while after laboratory investigations $42(23.9 \%)$ were diagnosed as RTI. Among 39 provisionally diagnosed RTI cases 13 (33.33\%) were diagnosed as PID, $10(25.64 \%)$ as mixed infection and 05 (12.82\%) as vulvovaginitis clinically. Among 42 confirmed RTI cases 16 (38.09\%) were confirmed as bacterial vaginosis, $04(9.52 \%)$ as trichomoniasis, $07(16.66 \%)$ as candidiasis, $06(14.28 \%)$ as mucopurulent cervicitis while $01(2.38 \%)$ was diagnosed as syphilis. Among 28 abortion cases 10 (35.71\%) developed RTI while among 38 MR cases 18 (46.36\%) developed RTI. Among 34 IUD users 22 (64.7\%) developed RTI while for other contraceptive method users only 19 (15.8\%) developed RTI. Conclusion: Although the study was in a limited scale and the findings of it will not only serve as a guideline for taking preventive measures, but can be used as a basis for future research and planning.
\end{abstract}

Key words: Reproductive tract infection (RTI); vulvovaginitis; bacterial vaginosis.

\section{INTRODUCTION}

One element of reproductive health that directly concerns human well being is reproductive tract infections (RTI). Women and men are vulnerable to a variety of RTIs, but the burden of problems is very unequally divided between the two sexes. The prevalence of RTIs is due to non availability of and inaccessibility to health services and social and cultural prejudices and practice. WHO has estimated that over 200 million RTIs occur every year among women in developing countries due to sexually transmitted pathogens ${ }^{1}$.

Each year 1.3 million women die of reproductive health problems that are largely preventable 2 . The magnitude of the problem of RTIs is a growing public health concern in developing countries. Until recently, the researchers, planners and policy implementers have paid little attention to the problems of RTIs in Bangladesh ${ }^{3}$.

Now, considering the widespread nature of RTIs, their multi-dimensional effect on child survival, family planning, safe motherhood, women's status and empowerment as well as the impending threat of an HIV/AIDS epidemic, collecting a range of epidemiological information and prevailing knowledge on RTIs is thought to be of high priority in Bangladesh ${ }^{4}$. Beside the pain and discomfort of acute illness, as a consequence of this infection, women often experience long term impairment of their reproductive health, some consequence, such as ectopic pregnancy. 


\section{MATERIALS \& METHODS}

The study was a clinic based "descriptive cross-section" type of study; it was carried out in the Gynaecology OPD of an urban clinic at Dhaka, Bangladesh during the period from Jul 2007 to Dec 2007. The study population consists of the women of reproductive age group (15-49 Years) attending in the OPD, with one or other disease conditions. A total of 1061 women reported sick in the OPD during the study period. Preliminary selection was done employing a standard selection criteria checklist. An "systematic purposive sampling" was followed as a sampling technique. The first ten patients of the day who reported sick to the OPD with any gynecological problems were taken as a sample. Thus a total of 184 patients were initially selected as potential respondents. Out of these 176 were willing to participate in the study. A data collecting questionnaire was developed on the basis of variable identified and the objective of the study. Data were collected from two sources (i) through the prescription sheets of the patient given by the attending gynecologist, (ii) through the face to face interviews of the respondents by the investigators and (iii) Laboratory investigation results. The completed questionnaires were checked for any omission, logical in consistencies, improbabilities and coding errors. Frequency distributions were used to provide the descriptive data on the prevalence and types of RTIs in the study population. Relationship between RTI and some selected socio-demographic and medical obstetric risk factors those were hypothesized to affect RTI prevalent were tested by running a series of cross tabulation separately. Statistical significance was tested appropriate probability level.

\section{RESULTS}

Among 1061 reported women at an urban clinic at Dhaka 176 participated in the study selected as systematic purposive sampling technique. All the respondents were of reproductive age group. Mean age 30.56 \pm 6.1. Among all 174 (98.86\%) were married (Table 1).

Table 1: Distribution of respondents by age and marital status $(n=176)$.

\begin{tabular}{lcc} 
Characteristics & Frequency of respondents & Percentage \\
Age in Year & & \\
$15-19$ & 02 & 1.1 \\
$20-29$ & 54 & 30.7 \\
$30-39$ & 100 & 56.8 \\
$40-49$ & 20 & 11.4 \\
Mean Age 30.56 Years & $\mathrm{SD} \pm 6.1$ years & \\
Marital Status & & \\
Currently Married & 174 & 96.6 \\
Not currently Married & 2 & 3.4 \\
\hline
\end{tabular}

Among 176 respondents provisionally RTI was found among 39 $(22.16 \%)$, while 32 (18.19\%) had symptomatic RTI described as vaginal discharge. Later on after laboratory investigation of all $42(23.9 \%)$ were confirmed as RTI (Table-2).
Table 2: Prevalence as per symptomatic and provisional diagnosis $(\mathrm{n}=176)$.

\begin{tabular}{lcccccc} 
Disease type & \multicolumn{2}{c}{ Symptomatic } & \multicolumn{2}{c}{ Provisional } & \multicolumn{3}{c}{$\begin{array}{c}\text { Confirmed (By lab } \\
\text { investigations) }\end{array}$} \\
& No & $\%$ & No & $\%$ & No & $\%$ \\
RTI & 32 & 18.19 & 39 & 22.16 & 42 & 23.9 \\
Non RTI & 144 & 81.81 & 137 & 77.84 & 134 & 76.1 \\
Total & 176 & 100 & 176 & 100 & 176 & 100 \\
\hline
\end{tabular}

Among 39 provisionally diagnosed RTI cases 13 (33.33\%) were clinically diagnosed as PID. Among others 10 (25.64\%) had mixed infection, $05(12.82 \%)$ had vulvovaginitis, 04 $(10.26 \%)$ had chronic cervicitis, $04(10.26 \%)$ with non specific itching, $02(5.12 \%)$ had dyspareunea and $01(2.57 \%)$ had genital ulcer (Table 3).

Table 3: Distribution of respondents by RTI related cases as per provisional diagnosis $(\mathrm{n}=39)$.

\begin{tabular}{lcc} 
RTIs & Frequency of respondents & Percentage \\
Vulvo -vaginitis & 05 & 12.82 \\
Mixed/Non specific Infection & 10 & 25.64 \\
Chronic cervicitis & 04 & 10.26 \\
PID & 13 & 33.33 \\
Genital Ulcer & 01 & 2.57 \\
Dyspareunea & 02 & 5.12 \\
Nonspecific itching & 04 & 10.26 \\
Total & 39 & 100 \\
\hline
\end{tabular}

Among 42 RTI cases which were diagnosed on laboratory investigations, $16(38.09 \%)$ were diagnosed as bacterial vaginosis, $04(9.52 \%)$ as trichomoniasis, $07(16.66 \%)$ as candidiasis, $06(14.28 \%)$ as mucopurulent cervicitis, 08 $(19.05 \%)$ as non specific vaginosis, $01(2.38 \%)$ as syphilis (Table 4).

Table 4: Distribution of respondents by types of non-RTI diagnosed as per laboratory methods $(n=42)$.

\begin{tabular}{lcc} 
Disease type & Respondents & Percentage \\
Bacterial vaginosis & 16 & 9.10 \\
Trichomoniasis & 04 & 2.28 \\
Candidiasis & 07 & 3.95 \\
Mucopurulent cervicitis & 06 & 3.40 \\
Non-specific vaginosis & 08 & 4.56 \\
Gonorrhoea & 00 & 0.00 \\
Syphilis & 01 & 0.57 \\
Total & 42 & 23.86 \\
\hline
\end{tabular}

Among 176 respondents, 28 (15.9\%) had the history of termination of pregnancy by abortion and $38(21.6 \%)$ had the history of MR. Among 28 abortion cases 10 (35.71\%) developed RTI while among 38 MR cases 18 (46.36\%) developed RTI. In 32 (21.82\%) among 148 women RTI was found who had no history abortion. While in 20 (14.79\%) respondents out of 138 were found with RTI who had no history of MR. The difference is statically significant $(\mathrm{P}=<0.01)$ (Table 5). 
Table 5: Distribution of respondents in relation to RTIs and termination of pregnancy in different modes $(n=176)$.

\begin{tabular}{ccccc} 
RTIs & \multicolumn{2}{c}{ Abortion } & \multicolumn{2}{c}{ MR } \\
& Yes & No & Yes & No \\
Yes & 10 & 32 & 18 & 20 \\
& $(35.71 \%)$ & $(21.82 \%)$ & $(46.36 \%)$ & $(14.79 \%)$ \\
& 18 & 116 & 20 & 118 \\
& $(64.29 \%)$ & $(78.18 \%)$ & $(52.64 \%)$ & $(78.4 \%)$ \\
Total & 28 & 148 & 38 & 138 \\
& $(15.9 \%)$ & $(84.1 \%)$ & $(21.6 \%)$ & $(78.4 \%)$ \\
\hline
\end{tabular}

Among 176 respondents $154(87.5 \%)$ were using different contraception methods. Among 154, 54 (35.06\%) used oral pill for contraception. Among other methods injection- 36 (23.38\%), condom- 10 (6.49\%), IUD- 34 (22.07\%), sterilization- 10 (6.49\%) and others-10 (6.49\%) (Table 6).

Table 6: Distribution of respondents by contraceptive method used. $(\mathrm{n}=176)$

\begin{tabular}{lcc} 
Contraception & Frequency of respondents & Percentage \\
None & 22 & \\
Oral Pill & 54 & \\
Injections & 36 & \\
Condom & 10 & \\
IUD & 34 & \\
Sterilization & 10 & 100 \\
Others & 10 & \\
Total & 176 & \\
\hline
\end{tabular}

Among 34 IUD users 22 (64.7\%) developed RTI while among 120 respondents using other methods developed 19 (15.8\%) RTI cases (Table-7).

Table 7: Distribution of respondents in relation to RTI and Contraceptive method.

$\mathrm{n}=154$

\begin{tabular}{lccc} 
RTIs & \multicolumn{2}{c}{ Contraception } & Total \\
& IUD & Others Methods & \\
Yes & $22(64.7 \%)$ & $19(15.8 \%)$ & $41(26.6 \%)$ \\
No & $12(35.3 \%)$ & $101(84.2 \%)$ & $113(73.4 \%)$ \\
Total & $34(100 \%)$ & $120(100 \%)$ & $154(100 \%)$ \\
\hline
\end{tabular}

Among 42 RTI cases 15 (35.71\%) used always washed clean cloths as sanitary material during menstruation, 24 (57.14\%) sometimes reuse the cloths, $01(2.38 \%)$ used commercial pads and $2(4.76 \%)$ used combination of all (Table- 8$)$.

Table 8: Distribution of respondents with RTI and use of sanitary materials during menstruation. $(\mathrm{n}=176)$

\begin{tabular}{llllll} 
RTIs & \multicolumn{4}{c}{ Sanitary Materials Used } & Total \\
& $\begin{array}{l}\text { Washed Clean } \\
\text { Cloths }\end{array}$ & $\begin{array}{c}\text { Not always } \\
\text { washed \& } \\
\text { reuse }\end{array}$ & $\begin{array}{c}\text { Commercial } \\
\text {-pads }\end{array}$ & Combination & \\
Yes & $15(16.7 \%)$ & $24(38.7 \%)$ & $1(7.1 \%)$ & $2(20 \%)$ & $42(23.9 \%)$ \\
No & $75(83.3 \%)$ & $38(61.3 \%)$ & $13(92.9 \%)$ & $8(80 \%)$ & $134(76.1 \%)$ \\
Total & $90(100 \%)$ & $62(100 \%)$ & $14(100 \%)$ & $10(100 \%)$ & $176(100 \%)$ \\
\hline
\end{tabular}

\section{DISCUSSION}

The RTIs are important health problems affecting women of reproductive age but often remain unexplored in the current service delivery system of the health services. It is not unusual for women to "suffer in silence" either because the RTIs are as common they feel it to normal or because they are shamed. ${ }^{5}$ This culture of silence around the issue of RTI related problems in our society also inhibits women to seek medical care. Besides, some of the RTIs are asymptomatic and complaints on these are nonexistent.

In a maternal morbidity study women were asked about the presence of symptoms of PID during their postpartum period. Over 83 percent of respondents did not report any symptoms of PID ${ }^{6}$. In our study $18.19 \%$ symptomatically present as RTI while $23.09 \%$ actually had RTI. Several community based studies have been conducted to find out the prevalence of RTI /STD among men and women. RTI has been found to range from $21.9 \%$ to $32.9 \%$ among married women of reproductive age $^{5,7}$. Wasserheit $\mathrm{JN}$ et al carried out a study at rural community of Bangladesh found $22 \%$ prevalence of symptomatic RTI ${ }^{8}$.

In our study $18.19 \%$ women symptomatically presented as RTI which was described as abnormal vaginal discharge by them. While in a larger study conducted by Bogarts $\mathrm{J}$ et al found $15 \%$ presented as abnormal vaginal discharge among 1879 women attending a basic healthcare clinic in Mirpur, Dhaka, from July 1996 to April $1998^{9}$.

After laboratory study we found 16 (38.09\%) were diagnosed as bacterial vaginosis, $04(9.52 \%)$ as trichomoniasis, $07(16.66 \%)$ as candidiasis, $06(14.28 \%)$ as mucopurulent cervicitis, $08(19.05 \%)$ as non specific vaginosis , $01(2.38 \%)$ as syphilis. None of the respondents was suffering from gonorrheal infection. Bogerts J, et al found $29 \%$ as bacterial pathogens, $21 \%$ as candidiasis, $2 \%$ as trichomoniasis while $0.5 \%$ had gonorrheal infection'. Hawkes $\mathrm{S}$ et al found $5.9 \%$ bacterial vaginosis, $6.7 \%$ candidal $\mathrm{spp}$ infection, $0.5 \%$ gonorrheal infection and $0.8 \%$ trichomoniasis ${ }^{10}$. While in a study among women living around track stand found 5.4\% gonorrheal infection and $13 \%$ syphilis ${ }^{11}$. This significant difference is probably due to high risk population for STIs in later study.

In our study among 28 abortion cases 10 (35.71\%) developed RTIs while among 38 MR cases 18 (46.36\%) developed RTIs. While Bogerts $\mathrm{J}$, et al found 14\% women developing RTIs among 592 women reported for pregnancy interruption ${ }^{9}$. The prevalence in more in MR cases possibly due to untrained persons conducting MR.

In our study it was found among 176 respondents 154 (87.5\%) were using different contraception methods. Among 154, 54 (35.06\%) used oral pill for contraception. condom- 10 (6.49\%) and IUD- 34 (22.07\%). Among the IUD user 64.7\% developed RTI while among other contraceptive method users $15.8 \%$ developed RTIs, A study conducted by Hussain et.al also found highest prevalence of RTIs among IUD users ${ }^{12}$. Hawkes et al also found $50 \%$ prevalence of RTIs among IUD users ${ }^{10}$. 
All respondents in the study were habituated to use some sorts of protection during menstruations. RTIs were found more (57.14\%) prevalent among the women using used cloths for protection. Hakes et al found endogenous infections more commonly among Hindu than Muslim women. Whereas 31\% of Muslim women changed their sanitary protection more than three times a day, only $15 \%$ of Hindu women did so ${ }^{10}$. The study conducted by Naved et al in 1996 coincide with our result ${ }^{13}$.

\section{CONCLUSION}

Reproductive Tract Infection is an alarming concern for the reproductive health of urban women. This study has identified some statistical association which are not sufficient to define the at risk population. We recommend for further conducting large scale epidemiological studies to try to define causal association of RTIs.

\section{DISCLOSURE}

All the authors declared no competing interest.

\section{REFERENCES}

1. World Health Organization. (1994). "Youth and Sexually Transmitted Diseases" In The Health of Youth: Facts for Action.

2. Piet- Pelon NJ. "Reproductive Health in Bangladesh - A Sectoral Review” UNFPA, Dhaka, 1996; 83.

3. Ministry of Health and Family Welfare,GOB; Technical Standard and Service Delivery Protocol for Management of RTI/STD. Published - by QIP,AVSC International, Bangladesh.

4. Ministry of Health and Family Welfare ,GOB; Bangladesh AIDs prevention and control Programme Support Document -May 1997: "Strategic plan for the National AIDs Programme" of Bangladesh(1997-2001).

5. Mueller DR, Wasserheit J. “ The Culture of Silence” 1991;18(4);1-18.

6. Khan R.F, Akhter M.S, Akhter H.H, The impact of RTIs on Women"s Health and Lives in Bangladesh. An annotated Bibliography BIRPERHT.2001;124:1.

7. Hussain MA Rahman GS. Begum N. "Prevalence of RTI /STDs in a Rural area of Bangladesh". Save the Children (USA), Bangladesh;2000;38-39.

8. Wasserheit JN, Harris JR, Chakraborty J, Kay BA, Mason KJ. Reproductive tract infections in a family planning population in rural Bangladesh. Stud Fam Plan, 1989; 20(2): 69-80.

9. Bogaerts J, Ahmed J, Akhter N, et al. Title: Sexually transmitted infections among married women in Dhaka, Bangladesh: unexpected high prevalence of herpes simplex type 2 infection. Sex Transm Inf 2001; 77; 114-119.

10. Hawkes S, Morison L, Chakraborty J, et al. Reproductive tract infections: prevalence and risk factors in rural Bangladesh. Bulletin of the World Health Organization 2002; 80 (3):180-188.

11. Gibney L, Macaluso M, Kirk K, et al. Prevalence of infectious diseases in Bangladeshi women living adjacent to a truck stand: HIV/STD/hepatitis/ genital tract infections. Sex Transm Inf 2001; 77:344-350.

12. Hussain M, Amin A, Khan AH. Prevalence of STD in Rajshahi Medical College Hospital -A 5 years retrospective study. 1998;15(1):9-11.

13. Naved et.al, RTI/STD and Risky Sexual Behavior in the context of a Conservative Society. Working paper. Save the Children (USA), Dhaka .1996. 